\title{
Determinants of Adverse Drug Reactions Due to Tuberculosis Therapy in African Countries: a Systematic Scoping Review Protocol
}

Tasmiya Raúfo Irá ( $\nabla$ tasmiyaira@gmail.com )

University of Eduardo Mondlane Faculty of Science: Universidade Eduardo Mondlane Faculdade de Ciencias https://orcid.org/0000-0003-4584-6328

\section{Neusa Torres Tovela}

Centro de Investigação em Saúde de Manhiça: Centro de Investigacao em Saude de Manhica

\section{Elisa López Varela}

Barcelona Institute for Global Health: Instituto de Salud Global Barcelona

\section{Pravina Devi Laljeeth}

University of Kwazulu-Natal

Richard Beharilal

University of Kwazulu-Natal

Elizabeth Bolanle Ojewole

University of Kwazulu-Natal

\section{Protocol}

Keywords: Adverse drug reactions, tuberculosis therapy, antituberculosis regimen, African countries, risk factors

Posted Date: July 8th, 2021

DOI: https://doi.org/10.21203/rs.3.rs-678037/v1

License: (c) (i) This work is licensed under a Creative Commons Attribution 4.0 International License. Read Full License 


\section{Abstract}

Background: Adverse drug reactions (ADRs) remain a significant public health concern worldwide mainly in developing countries. Adverse drug reactions due to multi-drugs regimen for the treatment of tuberculosis (TB) have negative implications such as toxicity, leading to poor compliance and interruption of medication before completion. Understanding the profile of ADRs is critical to establishing specific strategies for early detection, prevention and clinical management. The present study aims to map the existing literature on the determinants of adverse drug reactions due tuberculosis therapy in African countries.

Methods and analysis: The scoping review will be guided by Arksey and O'Malley framework as well as recommendations by Levac et al., and Colquhoun et al. The search strategy will include searching electronic databases such as Cochrane library, PubMed, EBSCOhost (including Medline), ProQuest, Google scholar; CINAHL with Full Text and other sources such as World Health Organization (WHO) and governmental websites. Peer-reviewed literature and grey literature of primary and secondary studies with different study designs which addresses the main question will be included. Studies published within the period of 2000 to 2020 are eligible. The selection process will involve screening titles, abstracts, full texts. A standardized data charting form will be created in google forms to extract relevant information from the included studies. The NVIVO software version 12 will be used for thematic analysis of the studies to summarize the review findings. The quality of the included studies will be assessed using Mixed Method Appraisal Tool (MMAT) version 2018 and Authority Accuracy Coverage Objectivity Date Significance (AACODS) checklist (for grey literatures).

Discussion: The study anticipates finding and mapping relevant research studies in African context on determinants of adverse drug reactions due to tuberculosis therapy. The synthesis of this evidence base will help to identify research gaps and will serve as guidance for future research studies on strengthening of the pharmacovigilance system of ADRs and its clinical management in Africa. The study findings will be disseminated through the traditional academic platforms, such as peer-reviewed publications and presentations at relevant local and international conferences, symposiums, and seminars.

Systematic review registration: Prospero Registration Number: CRD42020203617.

\section{Background}

Tuberculosis (TB) remains a major public health concern worldwide, predominantly in developing countries (1). It is a communicable, contagious airborne infectious disease caused by Mycobacterium tuberculosis, a bacteria that affects lungs (pulmonary TB) but can also affect other sites (extra pulmonary TB) $(2,3)$ Approximately a quarter of the world's population is infected with $M$. tuberculosis and is thus at risk of developing TB (3). In 2018, an estimated 10 million people (5.7 million men, 3.2 million women, and 1.1 million children) had TB worldwide (4). African region contributed with $24 \%$ of the cases and is included among those regions with a high prevalence of the disease. This high prevalence entails the need for proper management and treatment so that its spread can be controlled (3). 
Tuberculosis treatment involves taking a minimum of 3 drugs for 6 to 9 months or up to 18 to 24 months in severe cases $(5,6)$. Drugs commonly used are classified as first-line (isoniazid, rifampicin, ethambutol, and pyrazinamide) and second-line drugs (group A - levofloxacin, moxifloxacin, gatifloxacin; group B amikacin, capreomycin, kanamycin, (streptomycin); group C - ethionamide/ prothionamide, cycloserine/ terizidone, linezolid, clofazimine, group D- pyrazinamide, ethambutol, High-dose isoniazid (D1), bedaquiline, delamanid (D2), p-aminosalicylic acid Imipenem-cilastatin, meropenem, amoxicillintlavulanate (Thioacetazone) $(\mathrm{D} 3))(7,8)$ The criteria to select the drugs and dosage of treatment depends on the characteristics of the patient (age, weight, medical condition), stage of tuberculosis (latent or disease) and drug susceptibility or resistance of tuberculosis (first-line is used for drug-susceptible tuberculosis and second-line for drug-resistant tuberculosis) $(6,8,9)$. The combination and side effects of these multiple drugs associated to the long-term exposure of the patient to them can lead to adverse drug reactions (ADRs) $(2,10)$, defined according to as WHO (11) "a noxious and unintended response to a drug, which occurs at doses commonly used for prophylaxis, diagnosis, therapy, or modification of physiological function."

Adverse drug reactions remain a significant public health concern worldwide (12) mainly in developing countries (1).They are among the major causes of morbidity, mortality and high cost of patient care $(8,13$, 14). The percentage of patients experiencing ADR during hospitalizations has been reported to range from $1.5-35 \%$. Also, the reported frequencies of hospital admissions attributed to ADRs vary from $0.1-16.8 \%$ (15). According to Prasad, Singh (16), worldwide estimates indicate that the prevalence of ADRs due to tuberculosis therapy ranges from $8-85 \%$ for first-line drugs, and $69-96 \%$ for second-line drugs. The differences between the two regimens can be justified by the fact that second-line drugs are substantially complex, long-term, and more toxic than first-line drugs (1).

Adverse drug reactions due to multi-drugs regimen of tuberculosis may lead to different clinical presentations such as hepatotoxicity, skin rashes, gastrointestinal disturbances, neurological and musculoskeletal disorders $(14,17)$. These ADRs may result in negative implications for the tuberculosis treatment, such as poor compliance, interruption of medication before completion, which ultimately can lead to therapeutic failures, emergence of drug-resistant microorganisms, increase of morbidity, reduced quality of life or death $(2,3,17,18)$. Due to the above implications, there is a demand for proper management of the ADRs in order to prevent its harm and achieve an effective therapeutic outcome (2, 16). Understanding the profile of ADRs is critical to establish specific strategies for its identification and response, including early detection, prevention and clinical management $(2,17,19,20)$.

There are many factors that are associated with ADRs due to tuberculosis treatment (16). According to Prasad, Singh (16) and Valadares, Carvalho (21), the main determinants of ADRs in patients receiving tuberculosis treatment are related to administrations of the drugs (dosage and time of the day at which medication is administered), and patient characteristics such as age, nutritional status, alcoholism behaviour, pre-existing diseases or dysfunctions like impaired liver and kidney function, and co-infection with Human Immunodeficiency Virus (HIV). Moreover, Sahu, Singh (22) reported that patient's genetic 
make-up is one of the influencers of ADRs in tuberculosis therapy, however, this determinant has not been fully investigated.

Several studies have reported the risk factors associated with adverse drug reactions due to tuberculosis drugs (10, 23-25). Chung-Delgado, Revilla-Montag (10) conducted a study in Lima-Peru and found that old age (over 40 years), anemia, medication for multidrug resistant tuberculosis, overweight/obesity status, and smoking history are independent risk factors associated with ADRs due to antituberculosis treatment. Furthermore, a study in Ethiopia concluded that alcoholism, smoking, and concomitant drug intake were independent predictors of ADRs (23). Han, Pang (24) in a study at China, found that ADRs were significantly associated with diabetes mellitus. Resende and Santos-Neto (25) conducted a systematic review which purposed to provide evidence on the risk factors associated with adverse reactions due to antituberculosis drugs. The outcome was that risk factors for adverse reactions to antituberculosis drugs included age (> 60 years), treatment regimens, alcoholism, anemia, and HIV coinfection, as well as sodium, and albumin deficiency. Notwithstanding the results of the above-mentioned studies highlight the need of the understanding the risk factors associated with adverse reactions to antituberculosis drugs which ultimately will lead to a better clinical management at health care facilities However, more studies need to be done to explore the regional or community-specific profile of the risk factors associated with ADRs due to tuberculosis therapy to strengthen and adjust pharmacovigilance and management of the ADRs.

The authors synthesized evidences on risk factors associated with adverse reactions to antituberculosis drugs from the selected studies that were reported from various countries across the world. Of the 16 articles reviewed by Resende and Santos-Neto (25), only one study was from Africa. The present systematic scoping review therefore aims to map the existing literature and summarize the information on determinants of ADRs due to TB therapy specifically in the African context. This review will provide an updated information about adverse drug reactions related to tuberculosis treatment particularly in Africa. To our knowledge, it will be the first scoping review in this knowledge area conducted in the African setting. This will focus on African continent as it is affected with high tuberculosis burden, and the highest prevalence of HIV infection that similarly to concomitant infection (TB-HIV) leads to weakness of immune system being the most critical risk factor for progression of active TB $(6,26,27)$. Moreover, Africa faces concerns such as overburdened health care systems with scarce resources, underdeveloped infrastructures; colliding epidemics of infectious and non-communicable diseases; widespread of concomitant use of traditional remedies, pharmacogenetics variants associated with the increased risk of ADRs, these problems potentially contribute to the burden of drug-related harm $(28,29)$.

The review findings will represent a snapshot of the African status of on the risk associated to adverse drug reactions due to tuberculosis therapy what will enable researchers to identify research gaps and guide future researches around this topic. Furthermore, the reliable data on risk factors to adverse drug reactions due to tuberculosis therapy in Africa provided by this study will increase the awareness and importance of this topic in health systems. In addition this will enable to inform decisions on surveillance, prevention and management of ADRs in one hand, and in another hand on clinical management of the 
disease itself. Therefore, these achievements can result in the improvements of therapeutic adherence and successful treatment and, as consequence, a decreased morbidity and mortality due to TB in Africa.

Considering the aim of the study the specific objectives are the following:

- To describe the types of adverse drug reactions precipitated by tuberculosis therapy;

- To identify the therapeutic regimen associated with the adverse drug reactions;

- To determine the differences of the risk factors associated with adverse drug reactions observed within the African countries.

\section{Methodology}

\section{Scoping review}

The current scoping review protocol was registered and published in the PROSPERO international prospective register for systematic reviews. It is registered under the following registration number: CRD42020203617.

Peer-reviewed literature and grey literature of primary and secondary studies with various study designs addressing the determinants of adverse reactions will be included in this scoping review. This review will follow the five stages of methodological framework defined by Arksey and O'Malley (30) and further described by Levac, Colquhoun (31) and Colquhoun, Levac (32). The framework will include: (1) identifying the research question; (2) identifying relevant articles; (3) study selection; (4) charting the data; (5) collating, summarizing and reporting the results and an additional step (6) methodological quality appraisal. The Preferred Reporting Items for Systematic Review and Meta-Analysis Extension for Scoping Reviews (PRISMA-ScR) checklist will be used as a guide for planning and documentation of the review methods (33) (see Additional file 1 - PRISMA ScR checklist and Additional file 2 PRISMA -P checklist).

\section{STAGE 1: IDENTIFYING THE RESEACH QUESTIONS}

\section{Main question}

The main research question that guide this study is:

- In patients who have received tuberculosis therapy in Africa, and who have presented adverse drug reactions, what are the existent evidences on determinants to this occurrence?

\section{Specific questions}

In the context of adverse drug reactions due to tuberculosis therapy in African countries, the study also intends to respond the following relevant research sub-questions:

- What types of adverse drug reactions have been reported in these countries?

- What antituberculosis therapeutic regimen may influence the occurrence of adverse drug reactions? 
- What are the differences in risk factors associated with adverse drug reactions among the African countries?

\section{Eligibility criteria of research questions}

Population Intervention Comparison Outcomes Settings (PICOS) framework (Table 1) will be used to determine the eligibility criteria of the research questions and to define searchable keywords.

Table 1 Framework for determining eligibility of research questions (PICOS).

\begin{tabular}{|ll|}
\hline Population & Patients who have received tuberculosis therapy \\
\hline Intervention & have presented adverse drug reactions \\
\hline Comparison & have not presented adverse drug reactions \\
\hline Outcome & $\begin{array}{l}\text { Primary outcome: determinants of adverse drug reactions; } \\
\text { Secondary outcomes: types of ADRs, main risk factor, therapeutic regimens influencing } \\
\text { ADRs, common risk factors related to ADRs among African countries. }\end{array}$ \\
\hline
\end{tabular}

\section{STAGE 2: IDENTIFIYING THE RELEVANT ARTICLES}

Primary, secondary research articles, and grey literature which address the main question will be included in this study. Literature searches will be conducted using Cochrane library, PubMed, EBSCOhost (including Medline), ProQuest, Google scholar; CINAHL with Full Text. Searches will be limited to articles published in English and Portuguese. World Health Organization (WHO) and governmental websites will also be examined for reports. The authors of eligible studies will be contacted if the article is not available for free access. Manual searches will be conducted by hand-searching reference lists of included studies, reviews, and/or meta-analyses. The search strategy will be adapted for each database and used a combination of mesh terms or keywords with Boolean operators (AND, OR). Search terms will include: Determinants/ Factors associated/Risk factors; adverse drug reactions; tuberculosis therapy/tuberculosis treatment, Africa/ African countries/ Individual name of each country (Figure 1 in appendix).The methodology for search in database will be piloted to assess its appropriateness.

\section{STAGE 3: STUDY SELECTION}

\section{Eligibility criteria of the studies}

\section{Inclusion criteria}

The review will include studies involving patients: 
- At any age;

- Diagnosed with any type of tuberculosis ( pulmonary or extrapulmonary or Latent Tuberculosis Infection (LTBI));

- Following any type of tuberculosis treatment ( $1^{\text {st }}$ or the $2^{\text {nd }}$ line of tuberculosis treatment, LTBI treatment, or other);

- Diagnosed as having: multiple drug resistant tuberculosis (MDR) or drug sensitive tuberculosis (DS);

- With any type of entry (new cases, relapses, and re-admission after cessation);

- At any stage of the treatment (initial or continuation phase);

- Patients with or without other medical conditions such as: HIV, anemia, diabetes;

The review will include studies that consider one of the following definition of ADRs:

- "a noxious and unintended response to a drug, which occurs at doses commonly used for prophylaxis, diagnosis, therapy, or modification of physiological function" WHO (11).

- "an appreciably harmful or unpleasant reaction, resulting from an intervention related to the use of a medicinal product, which predicts hazard from future administration and warrants prevention or specific treatment, or alteration of the dosage regimen, or withdrawal of the product"(34).

This will also include studies:

- describing or referring to analyses of the association between risk factors and occurrence of ADRs due to tuberculosis therapy;

- specifying as local of study an African country or Africa;

- presenting any type of study design;

- published in English and Portuguese languages;

- published from 2000 to 2020.

\section{Exclusion criteria}

The review will exclude studies which:

- Involve patients with HIV status positive following antiretroviral therapy.

- had described the analysis of association between risk factors and occurrence of ADRs solely due to therapy of other disease rather than tuberculosis

\section{Selection process}

The selection process will be done by two independent reviewers ( $\mathrm{TI}$ and NT), any discrepancy will be solved by third reviewers (EV and EO) through discussions until consensus is reached. The selection will involve three stages: 
- Stage 1: Screening titles of all articles identified by the search based on the key words. The researchers will conduct a comprehensive title screening; all studies that do not address the study's research question will be excluded along with all the duplicates. All studies selected for abstract and full text screening will be uploaded on Endnote $X 9$ software.

- Stage 2: Evaluation of abstract and full text considering the eligibility criteria;

- Stage 3: Solving possible disagreements and uncertainness.

The number of studies included and excluded at each stage, along with reasons for exclusion, will be presented in a PRISMA flow diagram (Figure 2). Copies of full articles for eligible studies will be obtained and maintained for data extraction. The authors of eligible studies will be contacted to access missing studies. The University of KwaZulu-Natal library service will also be used to access articles that are not available online as full articles. Full articles will be requested from the authors if articles are unavailable from the databases/sources. We conducted a pilot search using our keywords, and database results are attached in Table 2 in appendix.

Table 2 Results of pilot search

\begin{tabular}{|c|c|c|c|}
\hline Keyword search & $\begin{array}{l}\text { Date } \\
\text { of } \\
\text { Search }\end{array}$ & $\begin{array}{l}\text { Search } \\
\text { engine } \\
\text { used }\end{array}$ & $\begin{array}{l}\text { Number of } \\
\text { publications } \\
\text { retrieved } \\
\text { (results) }\end{array}$ \\
\hline 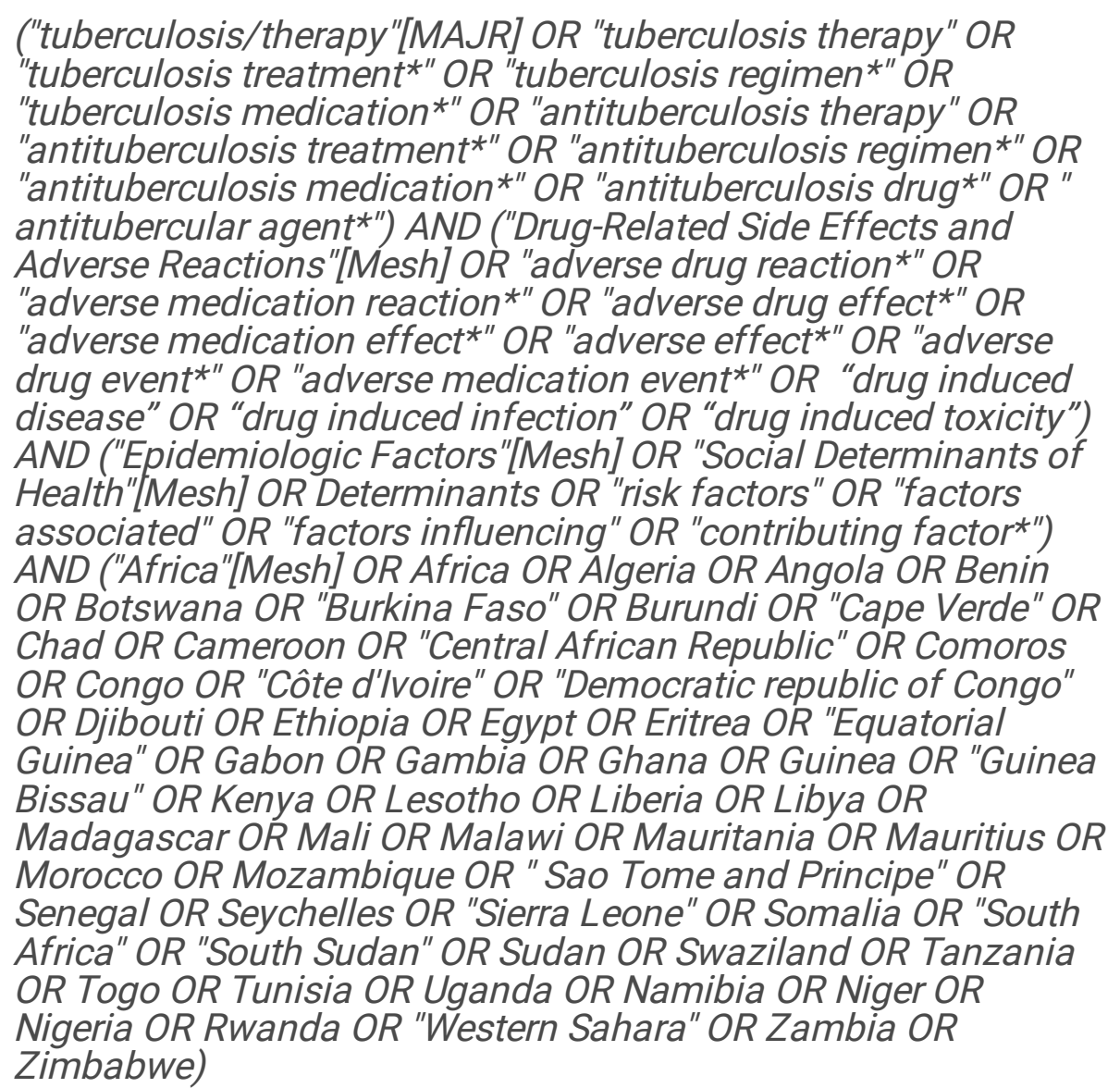 & $\begin{array}{l}31 \\
\text { May } \\
2021\end{array}$ & PubMed & 567 \\
\hline
\end{tabular}




\section{STAGE 4: CHARTING THE DATA}

A standardised data charting form will be created in google forms to extract data from the included studies. Prior to full data extraction, two reviewers (TI e NT) will pilot the data extraction form using a randomly selected sample of the included studies. Together with EV and EO any discrepancy will be addressed and the data form will be amended accordingly.

The data form will include general information about the study and specific information that allows to answer the research question (Table 3).

Table 3 Data charting form

$$
\begin{aligned}
& \text { - Title } \\
& \text { - Reference } \\
& \text { - Study location } \\
& \text { - Research question } \\
& \text { - Aims and Objectives } \\
& \text { - Demographics of participants } \\
& \text { - Recruitment method } \\
& \text { - Sampling method } \\
& \text { - Study design } \\
& \text { - Population } \\
& \text { - Data collection method } \\
& \text { - Data analysis } \\
& \text { - Intervention } \\
& \text { - Outcome }
\end{aligned}
$$

\section{STAGE 5: COLLATING, SUMMARIZING AND REPORTING THE RESULTS}

The data extracted from articles will be analysed using NVIVO software version 12 for thematic analysis. The themes will be derived from study outcomes. These will include the following: Adverse drug reactions 
types, adverse drug reactions description, therapeutic regimen influencing ADR, risk factors associations. Emerging themes and sub-themes will also be considered.

The resulting themes will be analysed and critically examined by their relationship to the research question. The researchers will analyse the meaning of the findings in relation to the aim of the study then will provide a narrative synthesis structured around the intervention (ADR description), target population characteristics (age, sex, TB therapeutic regimen, medical condition, alcoholism disorder), and the outcome ( risk factors associated to ADR).

\section{STAGE 6: METHODOLOGICAL QUALITY APPRAISAL}

The quality of each included study will be appraised using Mixed Method Quality Appraisal Tool (MMAT) version 2018. Two reviewers will perform the quality appraisal and will score the included studies independently using the two screening questions and a set of five questions each for the included qualitative studies, randomized controlled trial, non-randomized study, quantitative descriptive studies and mixed method studies as prescribed by the MMAT guidelines created by Hong, Pluye (36). Systematic reviews will be analysed under observational studies.

The tool will be utilized to examine the appropriateness of the aim of the study, adequacy and methodology, study design, participant recruitment, data collection, data analysis, presentation of findings, authors' discussions and conclusions.

Grey literature will be appraised using Authority Accuracy Coverage Objectivity Date Significance (AACODS) checklist (37).

\section{Discussion}

This scoping review aims to gather evidence on the determinants of adverse drug reactions due to tuberculosis therapy in African countries.

A systematic review on this topic was conducted in 2015 by Resende and Santos-Neto, in which worldwide available literature was critically included. This scoping review will provide updated information on the determinants of adverse drug reactions due to tuberculosis therapy, specifically in Africa. Attention is given to Africa because it is characterized by a high TB burden and risk factors associated and its vulnerability to have drug-related harms. Yet this area of research had received little attention in scientific research even though the increasing new cases of tuberculosis infection.

This specific investigation will help to know nature and extent of literature available about the purposed thematic in Africa, highlighting research gaps and so guide future research, and will enable to inform decisions on how to adjust the system of pharmacovigilance and management of the ADRs to prevent its harms and contribute to decrease morbidity and mortality due to TB. This review will also further describe the dynamics of adverse drug reactions due to tuberculosis treatment by exploring the characteristics of adverse drug reactions reported, the therapeutic regimen that may influence its occurrence, and the 
differences of risk factors associated with these adverse drug reactions among the African countries. This findings are very relevant as new tuberculosis regimens are appearing, and it is important to have benchmark for comparison, and standardized methods as we move forward, always focusing on reducing the toxicity during the treatment and decreasing morbidity and mortality.

This review will include all types of studies involving patients who experienced adverse reactions due to tuberculosis treatment in African countries. Considering this review's aim, this will obligatorily include studies that had described or referred to analyses of the association between risk factors and ADRs' occurrence due to tuberculosis therapy. Studies analysing risk factors to adverse drug reactions related to other diseases treatment will be excluded as they are not the interest of the review.

Articles published in Portuguese and English are privileged considering the fluency of the reviewers. Searching will be done excluding studies published before 2000 as the period before this year, African countries were still being intensively marked by developing national systems of pharmacovigilance on drug monitoring and by their adherence to WHO Program for International drug monitoring (38). This 20year of literature search will simultaneously allow us to briefly overview the past information and follow what has been done.

The review will be a scientific contribution to the field of pharmacovigilance of adverse drug reactions related to tuberculosis therapy in the African context, which interests the general public, healthcare professionals, researchers of pharmacovigilance, and policymakers. The dissemination plan of the results acquired include presentations at a research symposium, conferences, and submission a manuscript for a journal publication.

Limitations in this scoping review will arise due to the focus on mapping the breadth of studies instead of the depth of information, in order to identify research gaps. However, the chosen methodology is appropriate in addressing the review questions.

\section{Abbreviations}

ADRs: Adverse Drug Reactions; TB: Tuberculosis; PICOS: Population Intervention Comparison Outcomes Settings; MMAT: Mixed Method Quality Appraisal; AACODS: Authority Accuracy Coverage Objectivity Date Significance.

\section{Declarations}

\section{Acknowledgements}

The University of KwaZulu-Natal is acknowledged for providing free access to library services and licenses of the software programs required to conduct the research.

\section{Authors' contributions}


TRI conceptualized this study and prepared the draft manuscript under the supervision of EBO. All authors reviewed the draft manuscript, finalised the manuscript and approved it for submission to the journal.

\section{Funding}

None.

\section{Availability of data and materials}

Not applicable.

\section{Ethics approval and consent to participate}

Not applicable.

\section{Consent for publication}

The authors consent to publish the manuscript.

\section{Competing interests}

The authors declare that they have no competing interests.

\section{References}

1. Merid MW, Gezie LD, Kassa GM, Muluneh AG, Akalu TY, Yenit MK. Incidence and predictors of major adverse drug events among drug-resistant tuberculosis patients on second-line anti-tuberculosis treatment in Amhara regional state public hospitals; Ethiopia: a retrospective cohort study. BMC infectious diseases. 2019;19(1):286.

2. Naser S, Nandy M, Banu P, Banerjee A, Paul S, Podder I, et al. Adverse drug reaction monitoring through active surveillance of antitubercular therapy in an urban tertiary care center. Community Acquired Infection 2016;3(2):51-4.

3. WHO. Global tuberculosis report 2019. Geneva2019. 283 p.

4. WHO. Tuberculosis 2020 [Available from: https://www.who.int/news-room/factsheets/detail/tuberculosis. Accessed 20 Jun 2020.

5. Castro ATE, Mendes M, Freitas S, Roxo PC. Incidence and risk factors of major toxicity associated to first-line antituberculosis drugs for latent and active tuberculosis during a period of 10 years. J Revista Portuguesa de Pneumologia. 2015;21(3):144-50.

6. CDC. Treatment for TB Disease 2016 [Available from: https://www.cdc.gov/tb/topic/treatment/tbdisease.htm. Accessed 20 Jul 2020.

7. van der Werf T, Caminero J, Scardigli A, Tadolini M. Treatment of drug-susceptible and drug-resistant tuberculosis. Tuberculosis. 82: ERS Monograph, European Respiratory Society 2018. p. 
8. Venkateswarlu K, Tiwari K, Mamatha E, SaiVivek, P. S. Study of adverse drug reactions in tuberculosis patients. J Pharma Res. 2017;6:61-5.

9. WHO. Guidance for national tuberculosis programmes on the management of tuberculosis in children. Geneva, Switzerland: World Health Organization; 2014.123

10. Chung-Delgado K, Revilla-Montag A, Guillen-Bravo S, Velez-Segovia E, Soria-Montoya A, Nunez-Garbin $A$, et al. Factors associated with anti-tuberculosis medication adverse effects: a case-control study in Lima, Peru. PLoS One. 2011;6(11):e27610.

11. WHO. The importance of pharmacovigilance. United Kingdom2002. 48 p.

12. Khalil H, Huang C. Adverse drug reactions in primary care: a scoping review. BMC Health Services Research. 2020;20(1):5.

13. Chan SL, Ang X, Sani LL, Ng HY, Winther MD, Liu JJ, et al. Prevalence and characteristics of adverse drug reactions at admission to hospital: a prospective observational study. Br J Clin Pharmacol. 2016;82(6):1636-46.

14. Fei CM, Zainal H, Ali IAH. Evaluation of Adverse Reactions Induced by Anti-Tuberculosis Drugs in Hospital Pulau Pinang. Malays J Med Sci. 2018;25(5):103-14.

15. Pourpak Z, Fazlollahi MR, Fattahi F. Understanding adverse drug reactions and drug allergies: principles, diagnosis and treatment aspects. Recent patents on inflammation \& allergy drug discovery. 2008;2(1):24-46.

16. Prasad R, Singh A, Gupta N. Adverse drug reactions in tuberculosis and management. Indian J Tuberc. 2019;66(4):520-32.

17. Shareef JNUPBM. A Study on Assessment of Adverse Drug Reactions in Patients with Tuberculosis in a Tertiary Care Teaching Hospital. Journal of Applied Pharmaceutical Science. 2018;8(04):099-104.

18. Honnaddi UC, Honnaddi M, Tharangini S, Hossain T, Somani R. Adverse Drug Reactions to First Line Anti-Tubercular Drugs-A Pharmacovigilance Study. International Journal of Pharmacological Research. 2016;6 (2):51-4.

19. Charumathi A, Suganthi S, Sathish KR, Babisha J, Sankar C. A Review on Management of Common ADRS of Antitubercular Drugs. International Journal of Pharmaceutical Sciences Review and Research. 2019;58(2):60-4.

20. Imam F, Sharma M, Khayyam KU, Al-Harbi NO, Rashid MK, Ali MD, et al. Adverse drug reaction prevalence and mechanisms of action of first-line anti-tubercular drugs. Saudi Pharm J. 2020;28(3):316-24.

21. Valadares RMC, Carvalho WdS, Miranda SSd. Association of adverse drug reaction to antituberculosis medication with quality of life in patients in a tertiary referral hospital Journal of the Brazilian Society of Tropical Medicine. 2020;53:1-6.

22. Sahu RK, Singh K, Subodh S. Adverse Drug Reactions to Anti-TB Drugs: Pharmacogenomics Perspective for Identification of Host Genetic Markers. Current drug metabolism. 2015;16(7):538-52.

23. Bezu H, Seifu D, Yimer G, Mebrhatu T. Prevalence and risk factors of adverse drug reactions associated multidrug resistant tuberculosis treatments in selected treatment centers in Addis Ababa 
Ethiopia. Journal of Tuberculosis Research. 2014;2: 144-54.

24. Han XQ, Pang Y, Ma Y, Liu YH, Guo R, Shu W, et al. Prevalence and Risk Factors Associated with Adverse Drug Reactions among Previously Treated Tuberculosis Patients in China. Biomed Environ Sci. 2017;30(2):139-42.

25. Resende LS, Santos-Neto ET. Risk factors associated with adverse reactions to antituberculosis drugs. Jornal brasileiro de pneumologia : publicacao oficial da Sociedade Brasileira de Pneumologia e Tisilogia. 2015;41(1):77-89.

26. Bruchfeld J, Correia-Neves M, Kallenius G. Tuberculosis and HIV Coinfection. Cold Spring Harb Perspect Med. 2015;5(7):a017871.

27. MacNeil A, Glaziou P, Sismanidis C, Maloney S, Floyd KJM, Report MW. Global epidemiology of tuberculosis and progress toward achieving global targets-2017. 2019;68(11):263.

28. Mekonnen AB, Alhawassi TM, McLachlan AJ, Brien J-aE. Adverse Drug Events and Medication Errors in African Hospitals: A Systematic Review. Drugs - Real World Outcomes. 2018;5(1):1-24.

29. Mouton JP, Njuguna C, Kramer N, Stewart A, Mehta U, Blockman M, et al. Adverse Drug Reactions Causing Admission to Medical Wards: A Cross-Sectional Survey at 4 Hospitals in South Africa. Medicine. 2016;95(19):e3437.

30. Arksey H, O'Malley L. Scoping studies: towards a methodological framework. International Journal of Social Research Methodology. 2005;8(1):19-32.

31. Levac D, Colquhoun H, O'Brien KK. Scoping studies: advancing the methodology. Implementation science : IS. 2010;5:69.

32. Colquhoun HL, Levac D, O'Brien KK, Straus S, Tricco AC, Perrier L, et al. Scoping reviews: time for clarity in definition, methods, and reporting. Journal of clinical epidemiology. 2014;67(12):1291-4.

33. Tricco AC, Lillie E, Zarin W, O'Brien KK, Colquhoun H, Levac D, et al. PRISMA Extension for Scoping Reviews (PRISMA-ScR): Checklist and Explanation. Annals of internal medicine. 2018;169(7):467-73.

34. Edwards IR, Aronson JK. Adverse drug reactions: definitions, diagnosis, and management. Lancet (London, England). 2000;356(9237):1255-9.

35. Moher D, Liberati A, Tetzlaff J, Altman DG. Preferred reporting items for systematic reviews and metaanalyses: the PRISMA statement. 2009;339:b2535.

36. Hong QN, Pluye P, Fàbregues S, Bartlett G, Boardman F, Cargo M, et al. MIXED METHODS APPRAISAL TOOL (MMAT) VERSION 2018 User guide 2018 [Available from: http://mixedmethodsappraisaltoolpublic.pbworks.com/w/file/fetch/127916259/MMAT_2018_criteriamanual_2018-08-01_ENG.pdf. Acessed 02 Out 2020.

37. Tyndall J. AACODS checklist 2010 [Available from: https://dspace.flinders.edu.au/xmlui/bitstream/handle/2328/3326/AACODS_Checklist.pdf. Acessed 02 Out 2020.

38. Ampadu HH, Hoekman J, de Bruin ML, Pal SN, Olsson S, Sartori D, et al. Adverse Drug Reaction Reporting in Africa and a Comparison of Individual Case Safety Report Characteristics Between Africa 
and the Rest of the World: Analyses of Spontaneous Reports in VigiBase®. Drug safety. 2016;39(4):335-45.

\section{Figures}

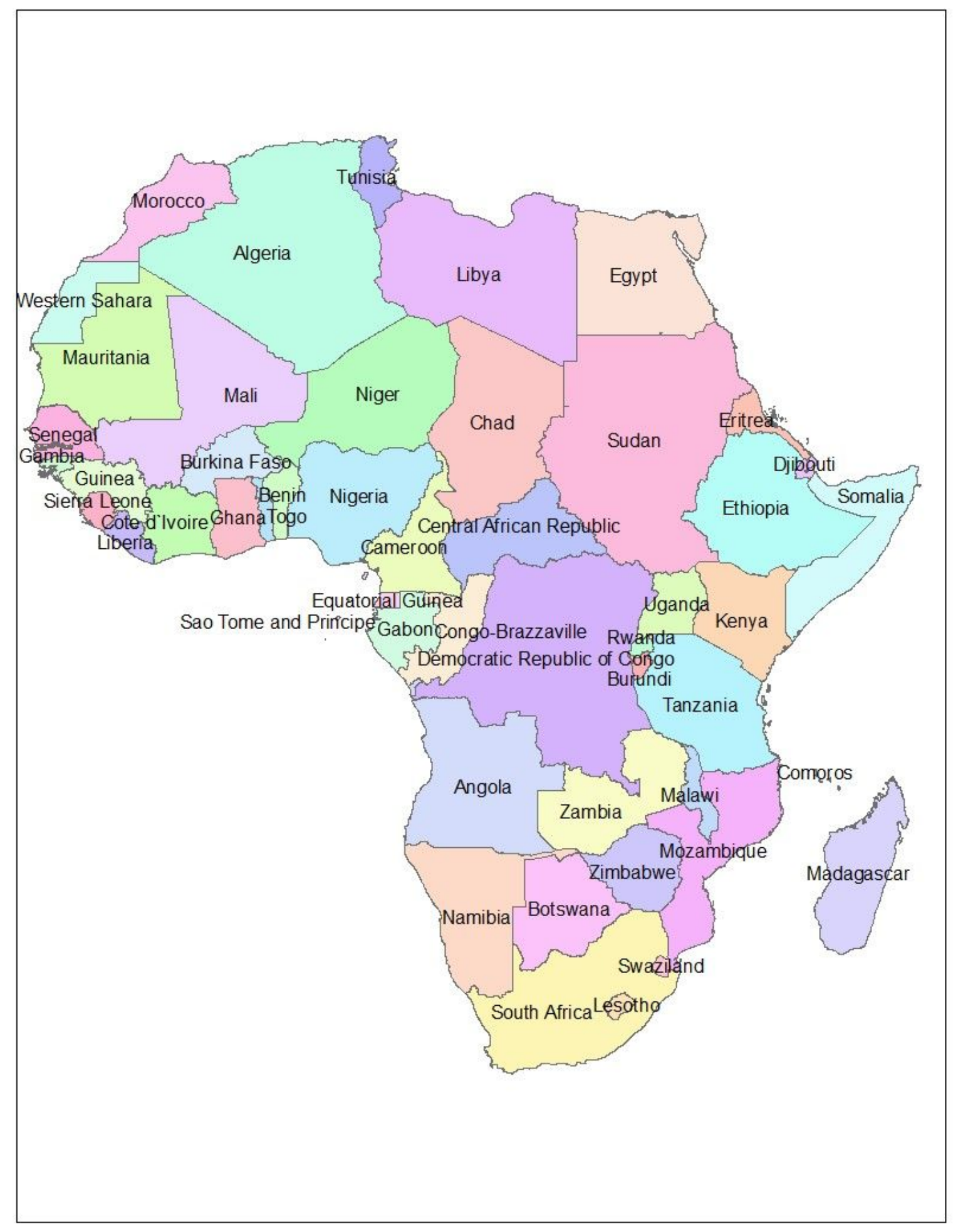

Figure 1 
Map of Africa indicating the geographical distribution of each country. Source: Created by the candidate using ArcMap 10.4
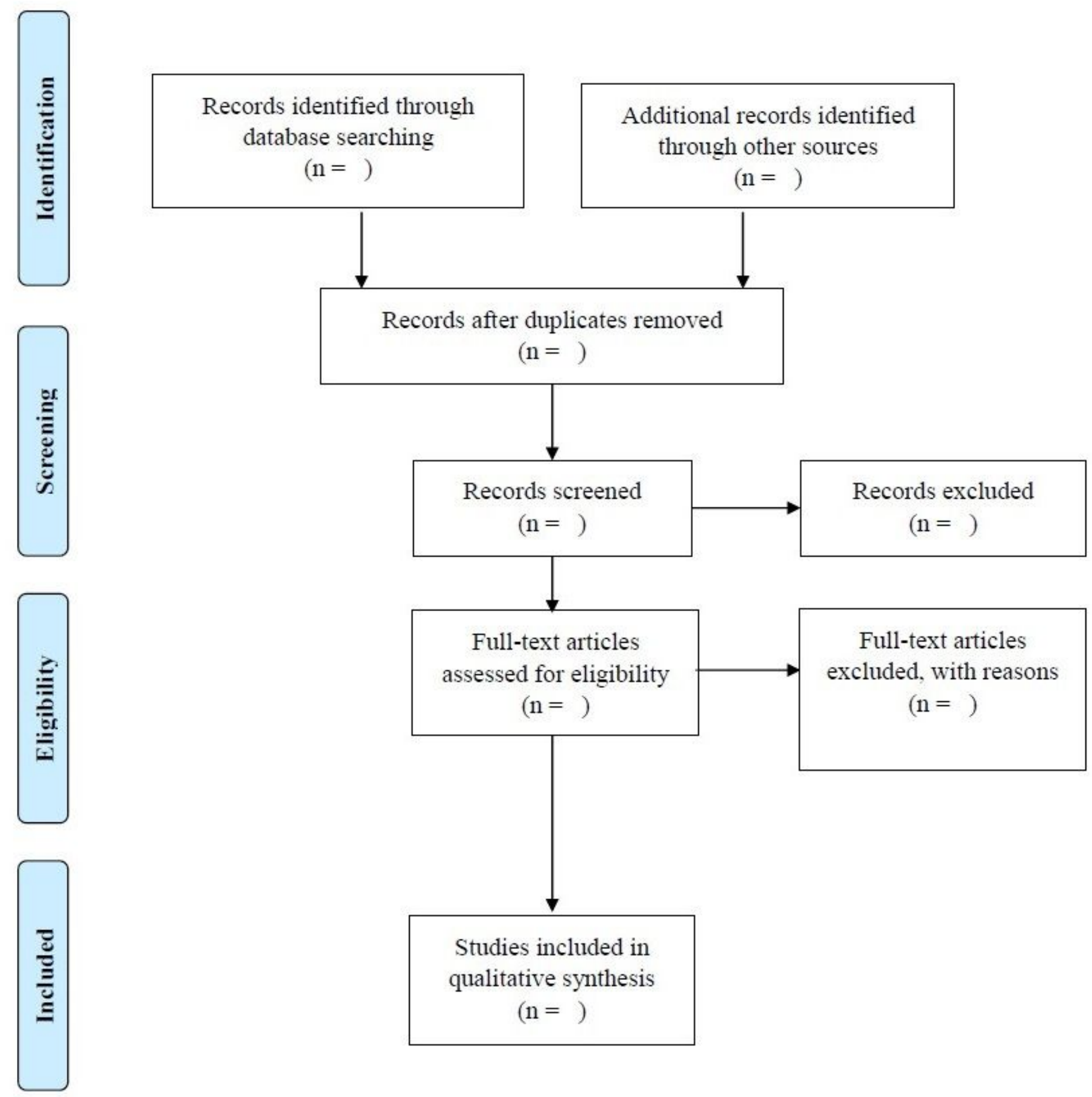

Figure 2

PRISMA flow diagram adapted from: Moher, Liberati (35), which will be used to demonstrate study selection process.

\section{Supplementary Files}


This is a list of supplementary files associated with this preprint. Click to download.

- Additionalfile1PRISMAScRchecklistcompletedpdf.pdf

- Additionalfile2PRISMAPchecklistcompletedpdf.pdf 